\title{
Errors Arising from Misalignment of the Measurement Location
}

\author{
Ross Castleton
}

May 1993

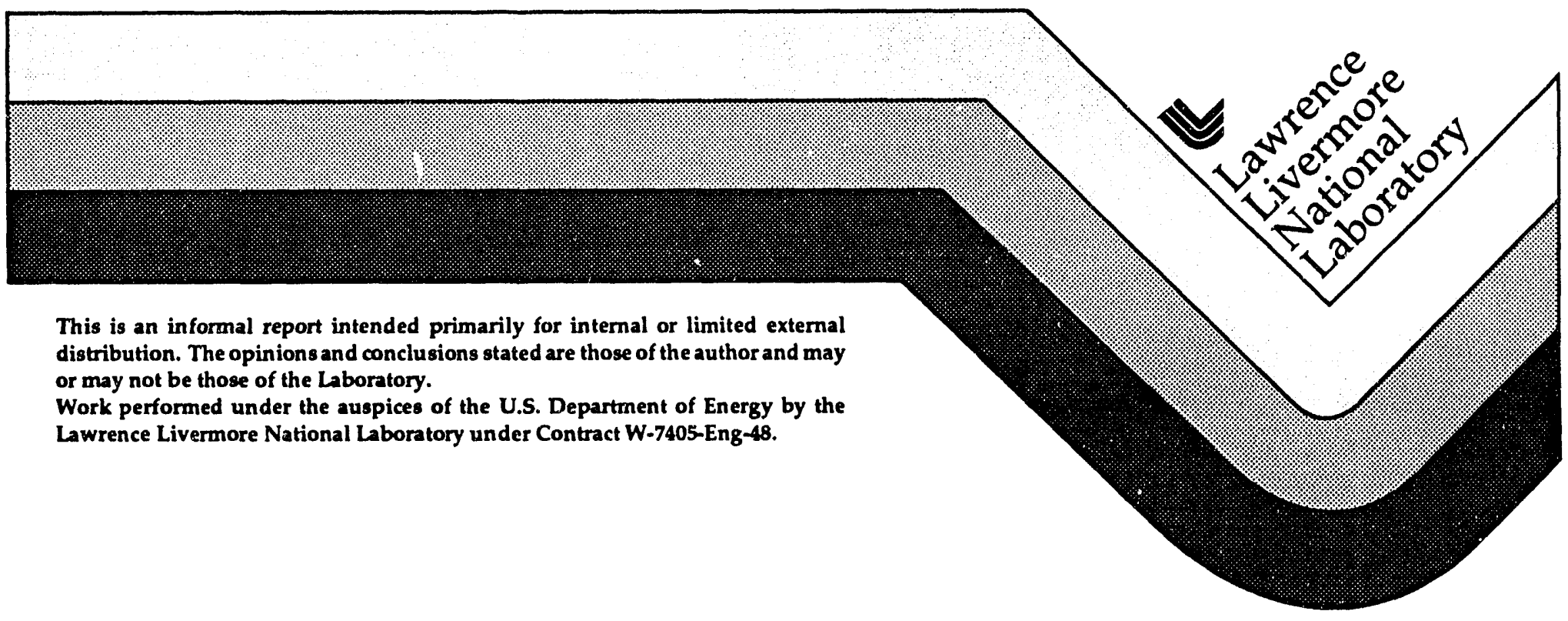




\section{Errors Arising from Misalignment of the Measurement Location}

An expression has been derived for the error resulting from a measurement location that is misaligned in the polar direction on the rurt profile. Such a misalignment could arise from a tilted part axis, or from a part being improperly centered, or from deviations of the actual part surface from the nominal part surface. These last two examples assume that the gage head probe is not aligned with the surface normal of the part.

Definitions:

1. The part profile is defined by the relationship $r=f(\theta)$.

2. The angle between the surface normal at the measurement point, and the radial direction at the measurement point is symbolized by " $\phi$ ".

3. The misalignment of the measurement location is symbolized by " $\Delta \theta "$.

Assumptions:

1. The misalignment of the measurement position, $\Delta \theta$, is small.

2. The radius of curvature of the part at the rneasurement position is not significantly different from the radial dimension at that point.

A few comments about the accompanying figure are in order. The figure shows a highly localized portion of the part surface, and the function $\mathrm{f}(\theta)$ is completely general. It is assumed that the desired measurement is $f(\theta)$, and the actual measurement is $f(\theta-\Delta \theta)$. Therefore the error that we are characterizing is

$$
\text { error }=\mathrm{f}(\theta-\Delta \theta)-\mathrm{f}(\theta) \text {. }
$$

In what follows, an expression is derived for this error which depends explicitly on the misalignment, $\Delta \theta$.

\section{Deriving the Expression}

From the figure (making use of the law of sines):

$$
\mathrm{d} / \sin (9()-(\phi-\Delta \theta))=(e+\delta) / \sin (\phi) .
$$

Rearranging terms:

$$
\mathrm{e}+\delta=\operatorname{error}+\delta+\varepsilon=\mathrm{d} \sin (\phi) / \cos (\phi-\Delta \theta)
$$

Using the assumption that $\Delta \theta$ is small, and the assumption that the radius of curvature of the part is not very different from the radial dimension of the part, $\delta$ and $\varepsilon$ will be very small, and $d$ can be replaced by $\Delta \theta f(\theta-\Delta \theta)$ :

$$
\text { error } \cong \Delta \theta \mathrm{f}(\theta-\Delta \theta) \sin (\phi) / \cos (\phi-\Delta \theta) \text {. }
$$

Thus the erro: in the radial measurement of the part surface depends, to the first order, on the misalignment. 
What remains to be addressed, is the justification of the approximations that have been used in deriving this expression for the error.

\section{The Approximations}

It is easily shown that $\delta$ is small. From the figure:

$$
\cos (\Delta \theta)=\mathrm{f}(\theta-\Delta \theta) /(\mathrm{f}(\theta-\Delta \theta)+\delta) .
$$

From this it follows that:

$$
\delta=\mathrm{f}(\theta-\Delta \theta)(1 .-\cos (\Delta \theta)) / \cos (\Delta \theta),
$$

and it can be shown that $|1 .-\cos (\Delta \theta)|$ is proportional to the square of $|\Delta \theta|$. See note 1 at the end of this paper. Because of this proportionality, for small $\Delta \theta, \delta$ is very small. Then because the radius of curvature of the part is assumed to be "close" to the radial dimension of the part, it follows that $\varepsilon$ is also very small.

The approximation of $\mathrm{d}$ by $\Delta \theta \mathrm{f}(\theta-\Delta \theta)$ is addressed next. From the figure:

$$
\mathrm{d}=\mathrm{f}(\theta-\Delta \theta) \tan (\Delta \theta)
$$

It follows that

$$
|\mathrm{d}-\Delta \theta \mathrm{f}(\theta-\Delta \theta)|=\mathrm{f}(\theta-\Delta \theta)|\sin (\Delta \theta)-\Delta \theta \cos (\Delta \theta)| / \cos (\Delta \theta) \text {. }
$$

It can be shown that $|\sin (\Delta \theta)-\Delta \theta \cos (\Delta \theta)|$ is proportional to the cube of $|\Delta \theta|$. See note 2 at the end of this paper. Thus the error in approximating the straight-line distance, $\mathrm{d}$, by the arc length, $\Delta \theta f(\theta-\Delta \theta)$, is not significant for small values of the misalignment, $\Delta \theta$.

\section{An Example}

Consider a part of general polar cross section, whose radial dimension at the elevation of measurement is 8 inches. Assume that the difference between the radial direction and the surface normal is 1) degrees at the measurement elevation. Further, assume that the hottom surface of the part is the measurement datum, and that the datum is tilted by 18.7 micro radians (this corresponds to 3()() micro inches of tilt if the radial dimension of the datum is also 8 inches). The formula for the error approximation yields the following result:

$$
\begin{aligned}
& \text { error } \cong \arcsin (.(0)() 3 / 16 .) 8 \sin (10 .) / \cos (10 .-\arcsin (.0()() 3 / 16 .)) \\
& =.(K)(K) 264 \text { inches }=26.4 \text { micro inches. }
\end{aligned}
$$

As a quick check on the approximations used to derive the formula, it is possible to compute the value of $\delta$ :

$$
\delta=8(1 .-\cos (\arcsin (.()()(1) 3 / 16 .))) / \cos (\arcsin (.()()) 3 / 16 .))
$$




$$
=.(0) 141 \text { inicro inches, }
$$

which is only .0(05\% of the 26.4 micro inch approximation.

The following table lists the results of four different misalignments of the above part.

$$
\text { Misalignment Error }
$$

$18.7 \mu$ radians $(3()) \mu$ inches $) \quad 26.4 \mu$ inches

$12.5 \mu$ radians $(20) \mu$ inches $) \quad 17.6 \mu$ inches

$6.3 \mu$ radians $(10) \mu$ inches $) \quad 8.8 \mu$ inches

$3.1 \mu$ radians ( $50 \mu$ inches) $\quad 4.4 \mu$ inches

\section{Notes}

The following pair of notes deal with the approximations used during the derivation of the error expression.

Note 1: In order to show that $|1 .-\cos (\Delta \theta)|$ is proportional to the square of $|\Delta \theta|$, consider the following approach. Expand the cosine using a Taylor series, factor out the common term, $\Delta \theta$ squared, and note that the remaining series is term-by term less than or equal to the series expansion of $\cosh (\Delta \theta)$, which is absolutely convergent.

Note 2: In order to show that $|\sin (\Delta \theta)-\Delta \theta \cos (\Delta \theta)|$ is proportional to the cube of $|\Delta \theta|$, expand both the sine and the cosine using Taylor series, multiply by the $\Delta \theta$, combine terms of like powers, factor out the common term, $\Delta \theta$ cubed, and note that the resultant series is term-by-term less than or equal to the series expansion of $\cosh (\Delta \theta)$, which is absolutely convergent. 


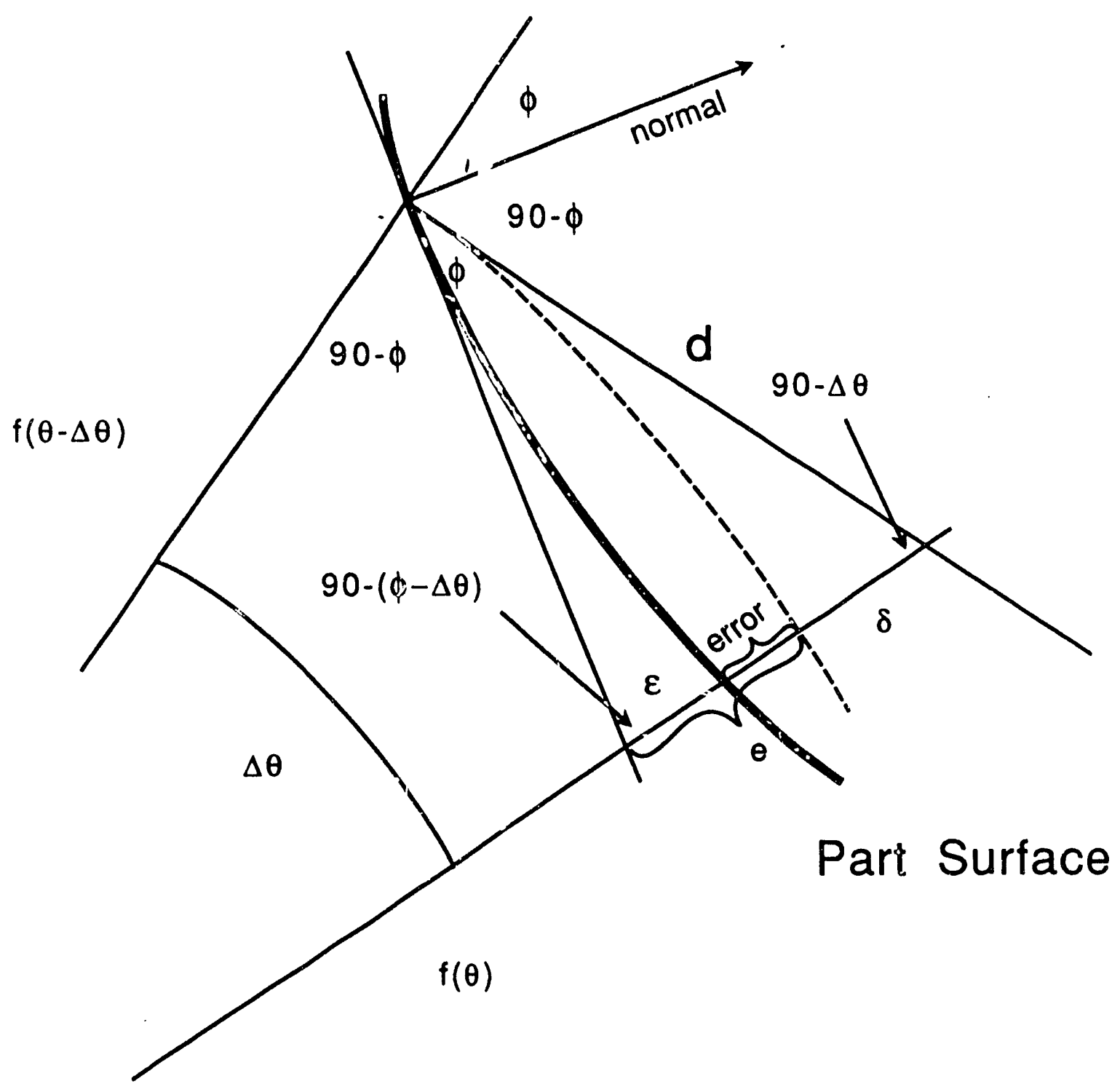



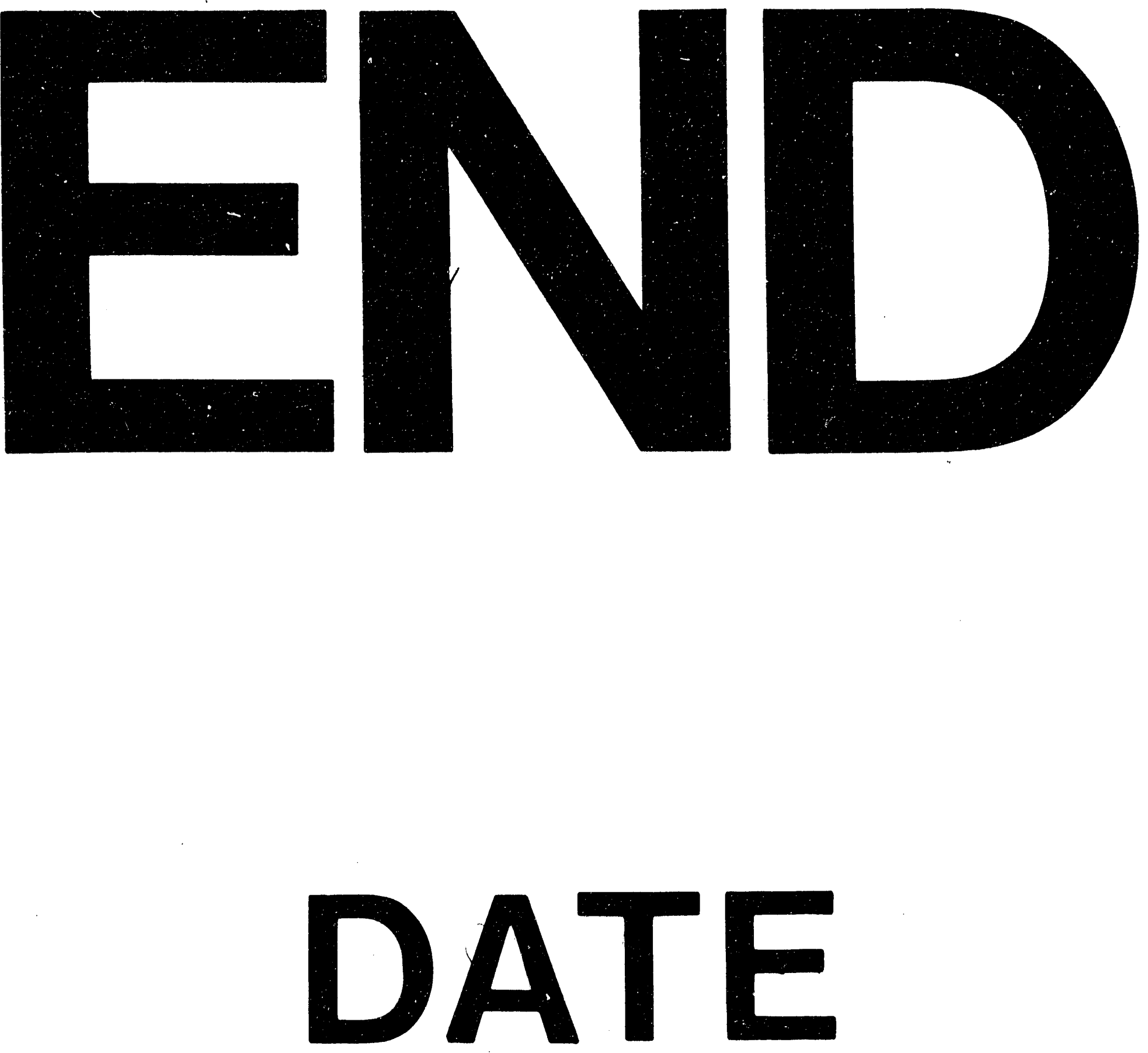

1
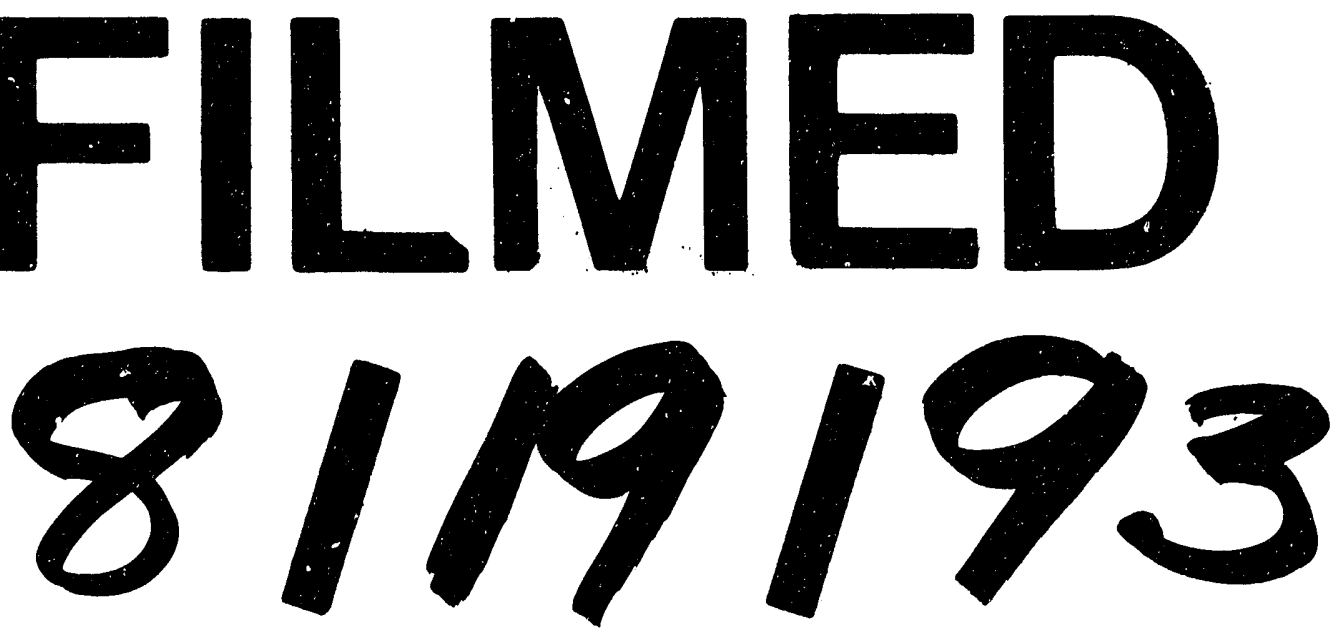
